\title{
Benchmarking beam alignment for a clinical helical tomotherapy device
}

\author{
John Balog and T. R. Mackie \\ TomoTherapy Incorporated, Madison, Wisconsin 53717 \\ and Department of Medical Physics, University of Wisconsin at Madison, Madison, Wisconsin 53706 \\ D. Pearson \\ TomoTherapy Incorporated, Madison, Wisconsin 53717 \\ Susanta Hui and Bhudatt Paliwal \\ Department of Medical Physics, University of Wisconsin at Madison, Madison, Wisconsin 53706 \\ Robert Jeraj \\ TomoTherapy Incorporated, Madison, Wisconsin 53717 \\ and Department of Medical Physics, University of Wisconsin at Madison, Madison, Wisconsin 53706
}

(Received 1 July 2002; accepted for publication 1 April 2003; published 29 May 2003)

A clinical helical tomotherapy treatment machine has been installed at the University of Wisconsin Comprehensive Cancer Center. Beam alignment has been finalized and accepted by UW staff. Helical tomotherapy will soon be clinically available to other sites. Clinical physicists who expect to work with this machine will need to be familiar with its unique dosimetric characteristics, and those related to the geometrical beam configuration and its verification are described here. A series of alignment tests and the results are presented. Helical tomotherapy utilizes an array of post-patient xenon-filled megavoltage radiation detectors. These detectors have proved capable of performing some alignment verification tests. That is particularly advantageous because those tests can then be automated and easily performed on an ongoing basis. (C) 2003 American Association of Physicists in Medicine. [DOI: 10.1118/1.1576395]

Key words: tomotherapy, commissioning dosimetry, beam alignment, multileaf collimator

\section{INTRODUCTION}

The first clinical helical tomotherapy ${ }^{1,2}$ treatment machine has been installed at the University of Wisconsin (UW) Comprehensive Cancer Center. The University of Wisconsin Tomotherapy Research Group and TomoTherapy Incorporated (Madison, WI) developed the hardware and software. It has been named Hi-ART for highly integrated adaptive radiotherapy. The precise, intensity-modulated radiotherapy treatments (IMRT) which this machine is capable of delivering require accurate patient-beam setup. That, of course, necessitates that the beam orientation be precisely known.

Tomotherapy utilizes a fan beam of radiation for treatment, which is inherently intensity modulated. The gantry rotates around the patient to treat from many angles. Axial tomotherapy extends the treatment region by sequential junctioning of the fan beam. ${ }^{3,4}$ Helical tomotherapy employs a synchronously rotating gantry and a translating couch. ${ }^{5}$ The Hi-ART system features a $6 \mathrm{MV}$ in-line linear accelerator. A photograph of a functioning tomotherapy machine is shown in Fig. 1. The tomotherapy coordinate system is shown in Fig. 2. A primary collimator (of tungsten) shapes the beam. This collimator defines a geometrical projection that is $40 \mathrm{~cm}$ wide in the $X$ or transverse direction by $5 \mathrm{~cm}$ wide in the $Y$ or patient inferior-superior (inf-sup) direction at an isocenter located $85 \mathrm{~cm}$ from the source. A single set of moveable (tungsten) jaws further collimates the $5 \mathrm{~cm}$ wide beam. This allows a variable width beam that is close to a $5 \mathrm{~cm}$ width in the inf-sup direction. These moveable jaws are shown in Fig. 3. The ends of the jaws are $23 \mathrm{~cm}$ from the source. A custom-made binary multileaf collimator (MLC) further collimates the beam in the transverse direction distal to the moveable jaws. This MLC contains 64 interspersed (tungsten) leaves. There are 32 leaves on each of two opposing sides (64 total) that slide past one another. The MLC is shown in Fig. 4. Each leaf therefore defines a beamlet that is $6.25 \mathrm{~mm}$ wide in the transverse direction at a distance of 85 $\mathrm{cm}$ from the source. The leaves are $10 \mathrm{~cm}$ thick in the beam $(Z)$ direction. The projected distance that the leaves traverse is more than $5 \mathrm{~cm}$ at the isocenter (they travel in the inf-sup direction). The leaves are binary (on or off) in the sense that the transit time from open to close is relatively short $(<25$ $\mathrm{ms})$. The leaves utilize an interlocking tongue-and-groove design to prevent a direct path through which the radiation can pass when adjacent leaves are closed. The tongue and the groove dimensions are both $0.30( \pm 0.03) \mathrm{mm}$ with a nominal overlap of $0.15( \pm 0.03) \mathrm{mm}$. The distal side of the MLC is approximately $40 \mathrm{~cm}$ from the source. A schematic diagram of the collimation is shown in Fig. 5.

A linear array CT detector resides on the rotating gantry opposite the source. This detector consists of 738 xenonfilled detectors. Each detector has a projected transverse width of $0.73 \mathrm{~mm}$ at the isocenter.

Alignment issues faced by clinical physicists during acceptance testing/commissioning and during routine quality assurance are addressed here. The tests are generally presented in the order in which they should be performed. The first are those that might necessitate moving the accelerator if the results were not satisfactory. Another concern was to de- 


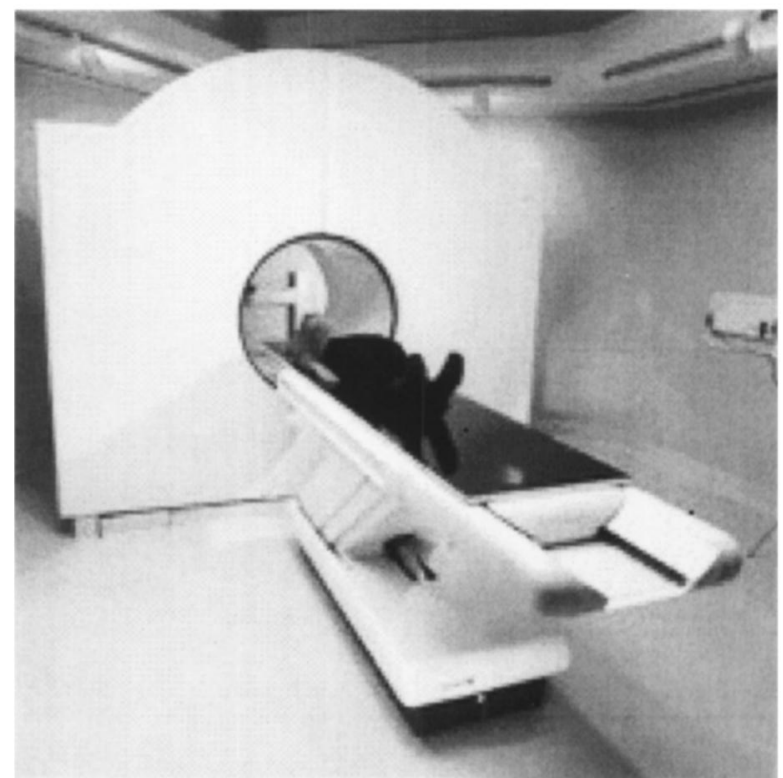

FIG. 1. This is a photograph of a helical tomotherapy unit. The slip-ring gantry and CT-like ring geometry is evident.

sign acceptance tests that required only those Hi-ART features that would be available at the time of alignment commissioning. The megavoltage computed tomographyimaging features would be useful for some tests, but will not be commissioned until after beam alignment, for example.

\section{MATERIALS AND METHODS}

Kodak EDR2 Ready-Pack film (Eastman Kodak Company, Rochester, NY) was used extensively for dose imaging. Radiographic film has become accepted for commissioning MLCs. ${ }^{6-11}$ EDR2 film is approximately linear in response to $600 \mathrm{cGy}$, and this extended dose range is better suited for tomotherapy dosimetry with its high output rate. The HiART accelerator produces about $1000 \mathrm{cGy}$ per minute for the calibration field size, $5 \mathrm{~cm}$ inf-sup width by $10 \mathrm{~cm}$ transverse width, at the isocenter distance $(85 \mathrm{~cm})$ at a depth of

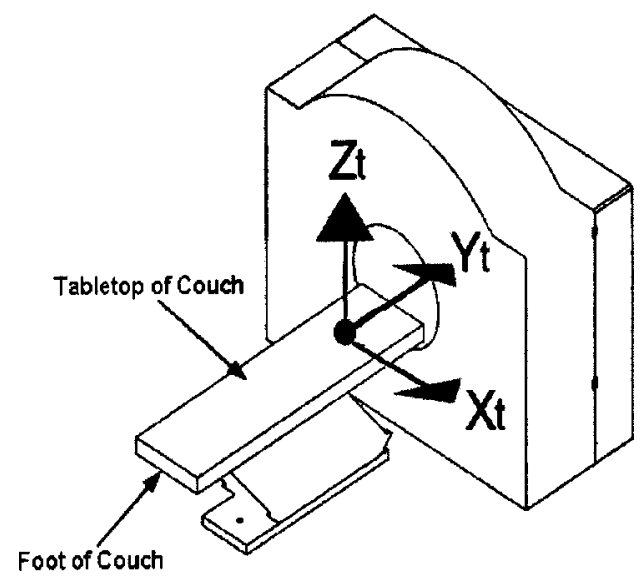

FIG. 2. This illustrates the helical tomotherapy coordinate system, which follows the International Electro technical Consortium (IEC) specification.
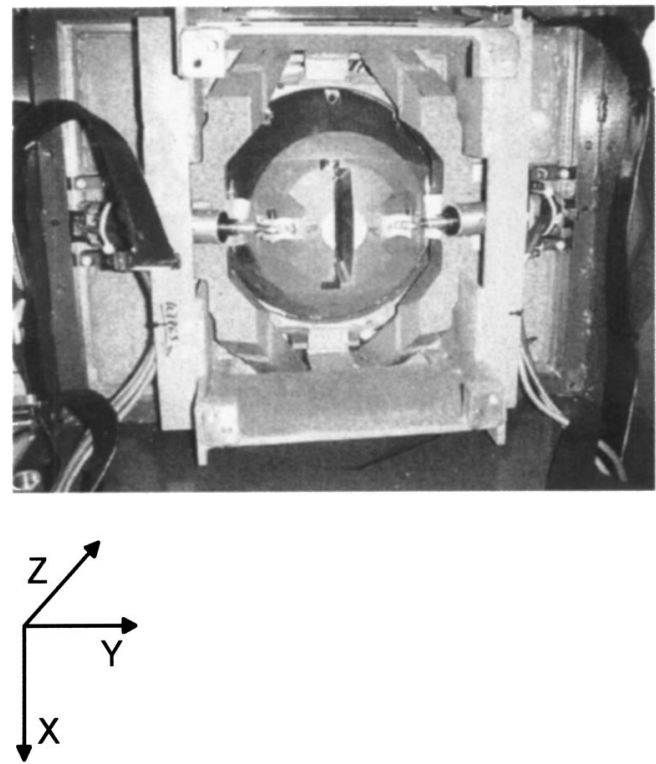

FIG. 3. This shows an image of the moveable jaws used to define the $Y$ axis or longitudinal field width.

$d_{\max }(1.5 \mathrm{~cm})$ in water. See the appendix for calibration details. Some films were exposed with the gantry only at $0^{\circ}$ (pointing down). Those films were at a depth of $1.5 \mathrm{~cm}$ with $2 \mathrm{~cm}$ of backscatter provided by custom made Solid Water slabs (Gammex Inc, Middleton, WI). Other films were exposed with the gantry at $0^{\circ}$ and then at $180^{\circ}$. Each of these films had $2.0 \mathrm{~cm}$ of build-up and $2.0 \mathrm{~cm}$ of backscatter. The film and solid water were positioned such that $10 \mathrm{~cm}$ of film and phantom overhung the end of the couch, for these situations. This allowed the radiation to undergo equal attenuation from both opposed gantry angles.

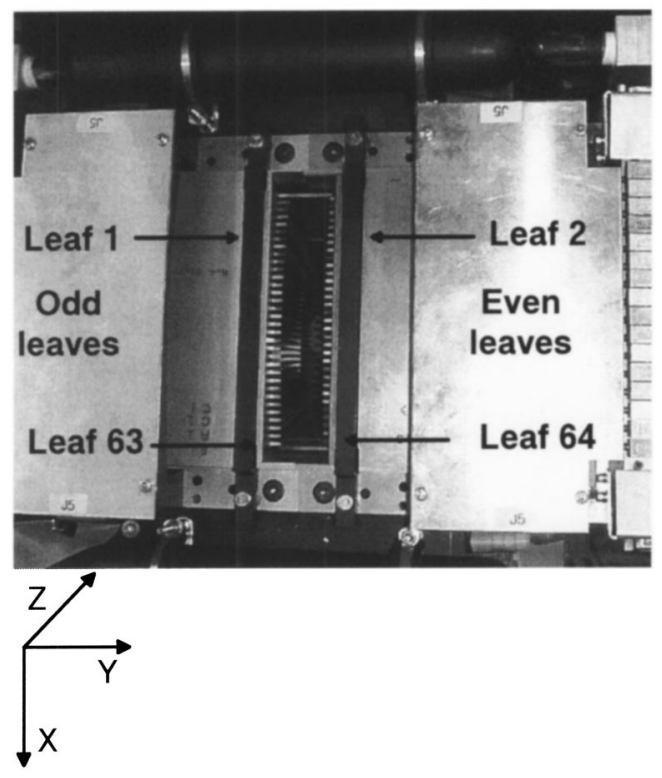

FIG. 4. This is an image of the binary MLC. 


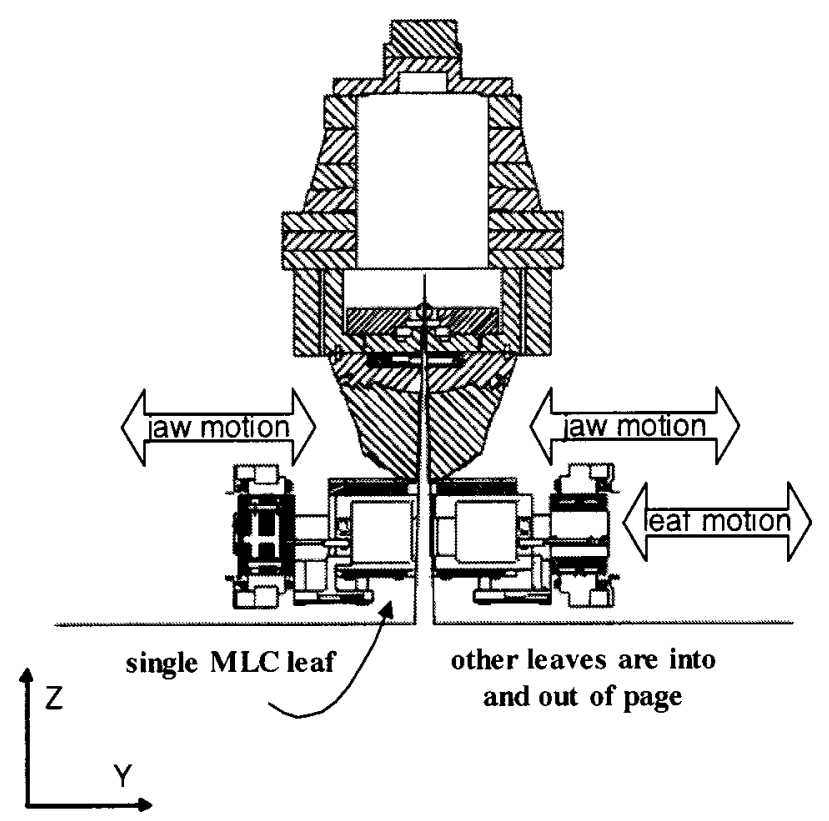

FIG. 5. This is a schematic diagram of the primary collimation, the moveable jaws, and a single MLC leaf.

All films were given a dose between 200 and 400 cGy to operate well within the linear range. They were scanned on a Howtek Multi-Rad 450 film digitizer (Hudson, NH). The measured digitization values were converted to dose via a known digitization value to dose calibration curve by exposing EDR2 film to known dose levels, and recording the corresponding digitization values. The calibration field setup was used. An ion chamber placed $2 \mathrm{~cm}$ below the film confirmed the dose. An appropriate percent depth dose correction factor was used.

Many of the tests require that the center of the slit beam be determined. This is defined as the halfway point between the full-width at half-maximum dose points. Custom analysis software was written in PV-Wave programming language (VNI Inc. Boulder, Colorado). This was done for both the digitized films and the data set recorded on the xenon exit detectors. Repeated use of the film and digitizer indicate that the full-width at half-maximum distance is accurate to within $\pm 0.2 \mathrm{~mm}$. This value was used for error analysis when appropriate. This uncertainty decreases to $\pm 0.1 \mathrm{~mm}$ when reanalyzing the same film.

\section{A. Source/primary collimator alignment}

The exact positioning of the source is critical due to the very small fields and the relatively short distance from the source to the primary collimator. The goal was to find the position of the jaws that maximized the output of the accelerator. Slight misalignments would be magnified and readily noticeable. The Hi-ART design allows a convenient method for verifying the source placement relative to the primary collimator along the $Y$ axis. The moveable jaws pivot about a point that is $50 \mathrm{~mm}$ behind the target position. The moveable jaws were asymmetrically shifted about their nominal center point such that the jaws-defined beam center swept across the physical source position. The moveable jaw offsets were swept from $-20 \mathrm{~mm}$ to $+20 \mathrm{~mm}$ at the isocenter plane in a series of discrete positions. The actual distance swept at the source location was $-2.5 \mathrm{~mm}$. This is equal to the -23 to $+23 \mathrm{~mm}$ shift at isocenter demagnified by $50 \mathrm{~mm} / 900 \mathrm{~mm}$, which is the pivot to source distance divided by the pivot to isocenter distance. All MLC leaves were open for each treatment procedure delivered for each static position of the moveable jaws. The moveable-jaws-defined $Y$-axis field width was kept constant at a relatively small distance of 4 $\mathrm{mm}$ projected to the isocenter. This increased the sensitivity of the test, since with a smaller jaw opening the transmitted fluence is a strong function of when the jaws-defined-centralaxis points toward the actual source center. The accelerator was physically moved based on these results to position the source as close as possible to the center of the primary collimator.

A custom-made ion chamber from Standard Imaging (Middleton, WI) was used for these alignment measurements. It is a cylindrical ion chamber with a $12 \mathrm{~cm}$ long collecting length and an $8 \mathrm{~mm}$ diameter. The long axis of the chamber was set parallel to the $Y$ axis at the isocenter. It was placed in a solid water miniphantom with a $3 \mathrm{~cm}$ diameter. The $4 \mathrm{~mm} Y$-axis field width strikes the chamber at different inferior-superior regions as a function of moveable-jaw shift position, but the detected signal was approximately invariant with ion chamber position, due to its uniform response. The central $5 \mathrm{~cm}$ of this chamber was irradiated as the jaws were shifted. The chamber response in this region is constant enough, within $1 \%$, such that each individual reading could be used without correction. This measurement is forgiving for small translation setup errors in any direction.

\section{B. Beam Y-axis misalignment}

The second alignment test verifies that the beam center is directed exactly parallel to the gantry rotation plane. Any $Y$-axis misalignment of the beam center could be detected by placing the EDR2 film well away from the gantry isocenter.

The film was placed below the axis of rotation so radiation directed from above had a relatively long distance to travel and therefore a relatively long distance through which to diverge. Next, the gantry was rotated $180^{\circ}$ and the film was irradiated again. The second exposure traveled less before it struck the film, so it diverged less. This concept is illustrated in Fig. 6. Any $Y$-axis misalignment of the beam center away from the gantry plane of rotation was readily apparent on the exposed image because the longitudinal centers of the two exposures were different.

The couch was dropped $25 \mathrm{~cm}$ below isocenter, and exposed through all leaves on one side of the MLC, 1-32, with the gantry at $0^{\circ}$. These were the left side or negative- $X$ side leaves with the gantry at $0^{\circ}$. The moveable jaws defined a 2 $\mathrm{cm}$ long $Y$-axis field width. The gantry was then rotated $180^{\circ}$, and the exposure was repeated using the same open-leaf configuration. Opening half of the MLC leaves for both exposures results in abutting images, that do not significantly overlap at the beam center. The films were processed, and the 


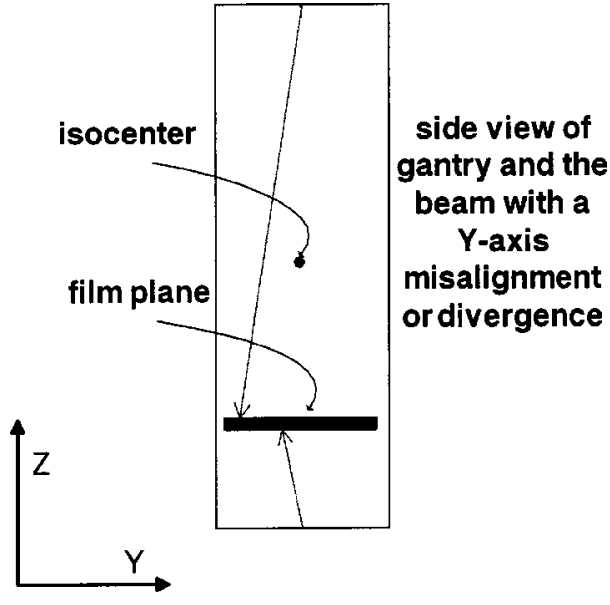

FIG. 6. This shows an illustration of the test for determining if the central axis of the beam has a $Y$-axis misalignment.

centers of each half-field exposure were independently determined. The resulting dose images are shown in Fig. 7. The centers of each half-field dose image were determined and compared. The moveable jaws were asymmetrically shifted until the beam center had a minimal $Y$-axis component.

The test just described only verifies that a specific jaw setting does or does not have a beam center with a $Y$-axis misalignment. This needs to be verified for other settings for the moveable jaws as well. An easy way to do this is to set a film at the isocenter and program a center set of leaves to open along with two other leaf sets positioned transversally away. The film is exposed using the $5 \mathrm{~cm} Y$-axis field width. Next two additional sets of leaves between the first set of previously open-leaf sets, are programmed to open. The moveable jaw opening is set to a clinical inf-sup field width, and the film is exposed again.

A film was placed at isocenter. The moveable jaw setting was set to the calibration inf-sup field width of $5 \mathrm{~cm}$. Leaves $11-18,29-36,47-54$ were opened and the film was exposed. The moveable jaws were narrowed to the clinical $Y$-axis $1 \mathrm{~cm}$ field width. Leaves $20-27$ and 56-63 were then opened, and the film was exposed again. The films were processed, and the centers of each leaf set were determined. The resulting dose images are shown in Fig. 8.

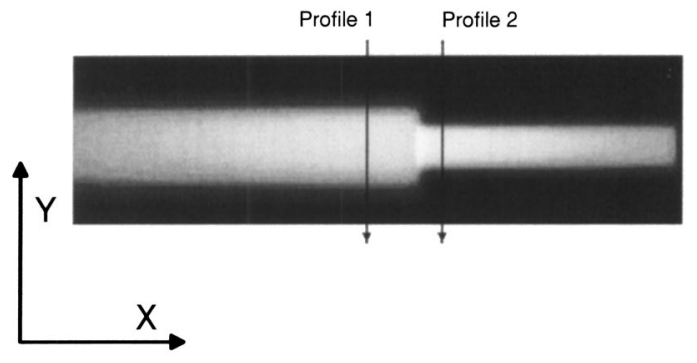

FIG. 7. This shows an illustration of the dose image that results when a film is placed $25 \mathrm{~cm}$ below the isocenter and irradiated through leaves $1-32$ with the gantry first at $0^{\circ}$ and second at $180^{\circ}$. Profiles taken across each half allow a central axis $Y$-axis misalignment to be quantified.

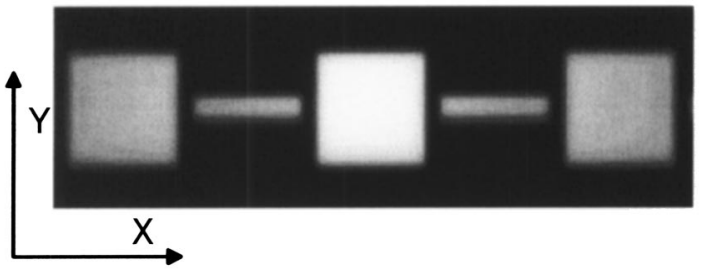

FIG. 8. This is the dose image that resulted from a double exposure to a film in which a series of leaves were opened for the calibration jaw setting and a separate set for another clinical jaw setting. Both jaws moved symmetrically if the centers of the dose blocks are the same.

A third criterion is that the moveable jaws must be parallel to the plane of gantry rotation. The slit beams will produce a criss-cross dose pattern upon gantry rotation if they are not. This concept is illustrated in Fig. 9. This condition was examined by another double exposure, gantry-rotation dose image of a film placed at the isocenter. All leaves were opened for both exposures. The first exposure was with the gantry at $0^{\circ}$ and the moveable jaws defined a $4 \mathrm{~cm} Y$-axis field width. The gantry-at- $180^{\circ}$ exposure used a $2 \mathrm{~cm} Y$-axis field width. The resultant dose image is shown in Fig. 10. $Y$-axis profiles were taken across the ends of this image and compared to determine the degree of jaw twist.

\section{MLC alignment}

The next test determines if the MLC is properly focused towards the source. One way to accomplish that is to utilize the tongue-and-groove/penumbral burring effect (T\&G-PB). The T\&G-PB effect has been described in the literature, ${ }^{12}$ but briefly it produces an energy-fluence dip between two sequentially opened leaves. This energy-fluence dip is minimized when leaves are maximally focused towards the source. The effect can be maximized for MLC-sourcealignment quality assurance by allowing only sequential

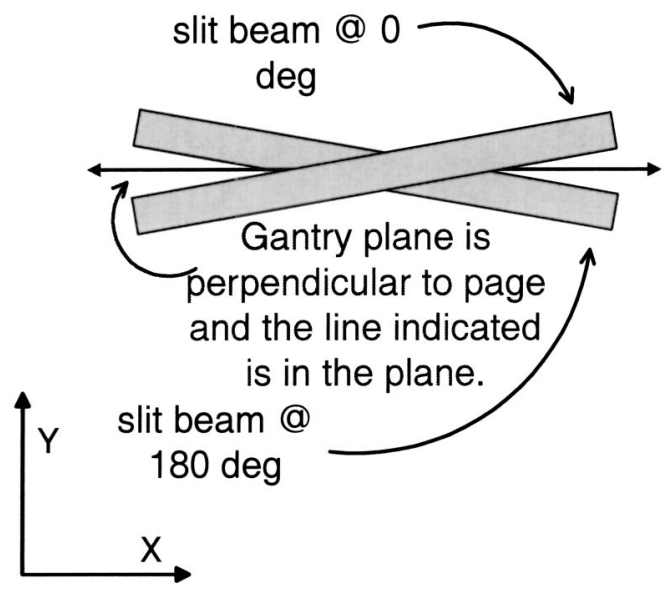

FIG. 9. This is an exaggerated illustration of the dose image that would result if the jaws were not perfectly aligned with the plane of gantry rotation. 


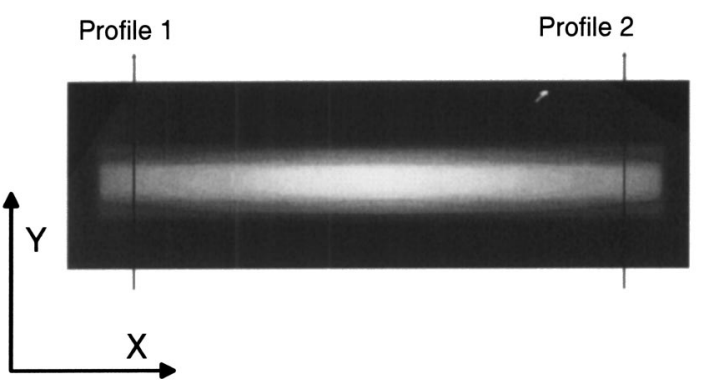

FIG. 10. This is a double exposure image of the gantry at $0^{\circ}$ and the gantry at $180^{\circ}$ image resulting from a $4 \mathrm{~cm}$ and a $2 \mathrm{~cm} Y$-axis field width. The two profiles were compared to determine the degree of jaw twist.

opening of adjacent leaves. (Note, the clinical delivery method minimizes sequential opening between adjacent leaves.)

A film was placed at isocenter with the gantry at $0^{\circ}$. Radiation was delivered in which every even leaf was open with all odd leaves closed, followed by every odd leaf open with all even leaves closed. This produced a series of $\mathrm{T} \& \mathrm{G}-\mathrm{PB}$ modulations. The resultant dose image is shown in Fig. 11. A transverse profile was measured across this image and analyzed for symmetry between the T\&G-PB peaks and valleys.

This same treatment procedure was delivered with the couch fully retracted from the bore. The xenon detector recorded the incident dose profile. The data were processed such that the all-even-leaf profile was added to the all-oddleaf profile. This determined how well the xenon detectors matched film for this experiment.

The MLC leaves could have a twist with respect to the plane of gantry rotation, as previously explained for the jaws, since the MLC mounting is independent from the jaws. The alignment was tested via a double exposure, gantry-rotation delivery to a film placed at the isocenter. Leaf 32 was open for each exposure taken at gantry angle $0^{\circ}$ and gantry angle $180^{\circ}$. The MLC center is between leaves 32 and 33 so the resultant two leaf-32 images should be adjacent, not superimposed. The moveable jaws were set to the $5 \mathrm{~cm} Y$-axis field width to increase the distance over which any MLC twist could be observed. The resultant dose image is shown in Fig. 12. Multiple profiles were taken across the dose image and compared.

The location of the MLC leaves with respect to the gantry center of rotation (COR) must be determined. A double exposure, gantry-rotation dose image from a film placed at the isocenter is used. The gantry-at- $0^{\circ}$ exposure had leaves 32

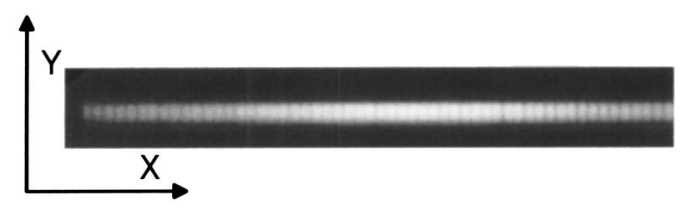

FIG. 11. This shows the dose image that results from an exposure through every odd leaf open followed by every even leaf open.

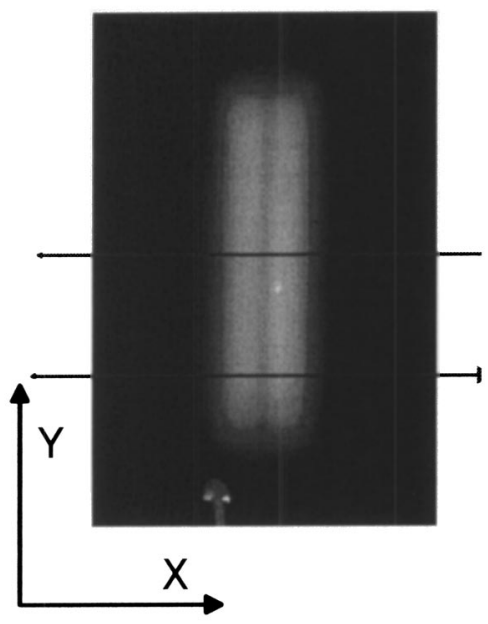

FIG. 12. This is the resultant dose image from a double exposure gantry rotation procedure. Leaf 32 was open for both exposures. The $Y$-axis field width was set to $5 \mathrm{~cm}$. Profiles taken along each line will be identical only if the MLC-leaf direction is exactly perpendicular to the gantry rotation plane.

and 33 and leaves 28 and 29 opened sequentially. The gantry-at- $180^{\circ}$ exposure had leaves 28 and 29 opened sequentially. The projection of the center of the MLC should lie at the leaf 32-33 T\&G-PB indication. The MLC center position relative to the gantry isocenter can be determined from the relative position of the T\&G-PB indications. The MLC/COR dose image is shown in Fig. 13.

The MLC/xenon detector relationship needs to be mapped, and to determine if that mapping is invariant with gantry position. This was done without film. A series of four T\&G-PB procedures were delivered at gantry angles $0^{\circ}$, $90^{\circ}, 180^{\circ}$, and $270^{\circ}$. Leaves 32 and 33 were opened sequentially for all exposures. The T\&G-PB artifact is clearly visible on the profile across the xenon detector data, and the detector channel that recorded it was noted for each profile. The consistency of this detector channel relationship was then determined as a function of gantry angle.

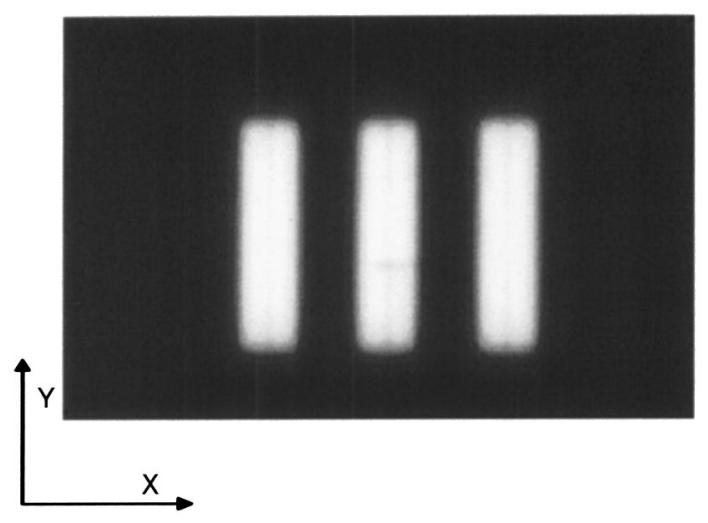

FIG. 13. This is the dose image that results from a double exposure gantry rotation in which leaves 32 and 33 and leaves 28 and 29 opened sequentially. 


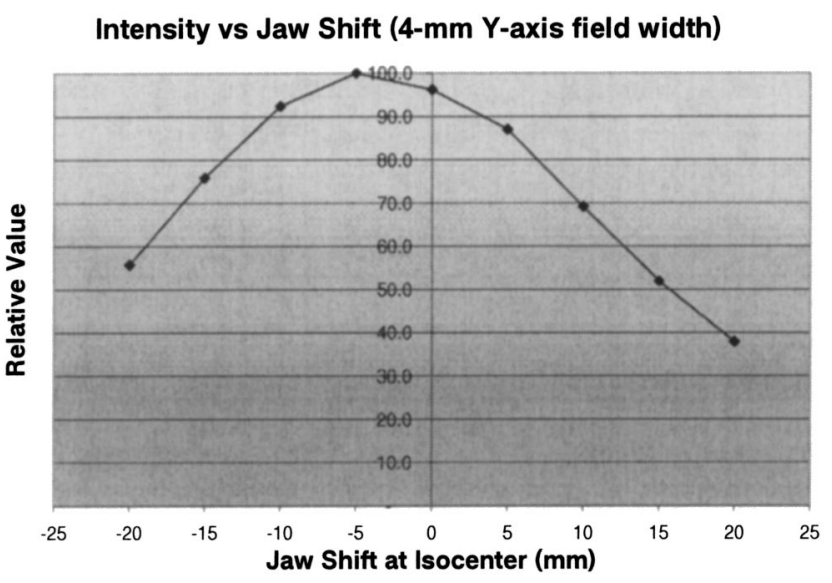

FIG. 14. This shows the relative intensity measured as a narrow jaw width was symmetrically shifted across the isocenter and source plane.

\section{Detector alignment}

The collimating face of the moveable jaws and the xenon detector array must be parallel. This can be determined by performing a series of treatment procedures in which the jaws are gradually closed until the signal on the xenon detectors starts to diminish. Manipulating the jaw-offset position did this. All detectors should see less radiation vs jaw size equally as the moveable jaw openings are decreased, if the detectors and jaws are perfectly aligned.

A detector profile was recorded for a $30.0 \mathrm{~mm}$ moveablejaw setting and a $7.5 \mathrm{~mm}$ offset, i.e., the projected center of the jaw opening at isocenter was shifted $7.5 \mathrm{~mm}$. This effectively had one jaw occluding part of the detectors while the other provided no beam blocking. A calibration reading normalized each offset reading. The calibration reading consisted of a $30.0 \mathrm{~mm}$ moveable-jaw setting with no shift. This removed the detector response variation as a function of detector position.

\section{RESULTS}

The majority of the dose images recorded on film were mathematically rotated so that the long axis of the slit beam was parallel with the imaging coordinate system. This enabled all center points (along the transverse plane) to lie in the same row.

\section{A. Source/primary collimator alignment}

The relative source intensity vs moveable-jaw shift position is shown in Fig. 14. The intensity was maximized with a jaw shift of negative $5 \mathrm{~mm}( \pm 2 \mathrm{~mm})$. This test reveals that the source was $0.3 \mathrm{~mm}( \pm 0.02 \mathrm{~mm})$ away from the center of the primary collimator, after accounting for the magnification of the movement at the isocenter. (The uncertainty was based on the source position prediction of negative $3 \mathrm{~mm}$ based on an interpolated full-width at half-maximum distance.)

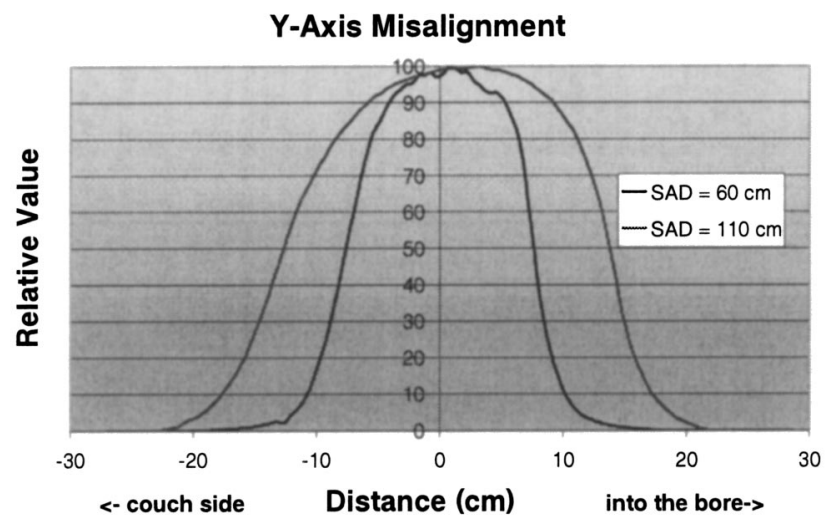

FIG. 15. This is a plot of the two profiles taken along the $Y$-axis misalignment dose image. The centers of the two images would be the same if there were no $Y$-axis misalignment of the central axis.

\section{B. Beam $Y$-axis misalignment}

The two profiles taken across the $Y$-axis misalignment dose images are shown in Fig. 15. The center of each profile can therefore be used to measure the $Y$-axis misalignment component of the beam center. The $Y$-axis location for the center of the top-directed beam equals the divergence slope multiplied by the distal SSD distance of $110 \mathrm{~cm}$. The $Y$-axis location for the center of the bottom-directed beam equals the divergence angle multiplied by the near distance of 60 $\mathrm{cm}$. The difference between the centers of the two exposures then equals the difference through which each beam diverges multiplied by the divergence slope,

Center difference $=$ divergence slope $^{*}($ far SSD

$$
\text { - near SSD), }
$$

Center difference $=$ divergence slope $* 50 \mathrm{~cm}$.

The angular value of the central axis misalignment is equal to the inverse tangent of the divergence slope,

Angular $Y$-axis misalignment of beam center

$$
\left.=\tan ^{-1} \text { (center difference } / 50 \mathrm{~cm}\right) \text {. }
$$

The center difference between the two profiles was measured to be $0.56 \mathrm{~mm}$. So, the actual angular $Y$-axis misalignment of the beam center $=\tan ^{-1}(0.056 / 50)=0.064^{\circ}$.

The $Y$-axis misalignment at the isocenter is computed to be equal to $(85 \mathrm{~cm} / 50 \mathrm{~cm}) * 0.56=0.95 \mathrm{~mm}$.

The two profiles taken across the varying moveable-jaw setting dose images are shown in Fig. 16. A shift in the location of the two centers indicates an unequal motion of the jaws with respect to the calibration field positions. The centers were offset by $0.5 \mathrm{~mm}$. The center of the clinical beam was $0.5 \mathrm{~mm}$ deeper into the bore.

The two profiles taken across the jaw/gantry-plane alignment dose image are shown in Fig. 17. The image was rotated based on the edges of the longer $Y$-axis field width. Each profile was $3 \mathrm{~cm}$ from the field edge, so the transverse distance between the profiles was $34 \mathrm{~cm}$. The field edges for the greater moveable-jaw setting match exactly, which indi- 


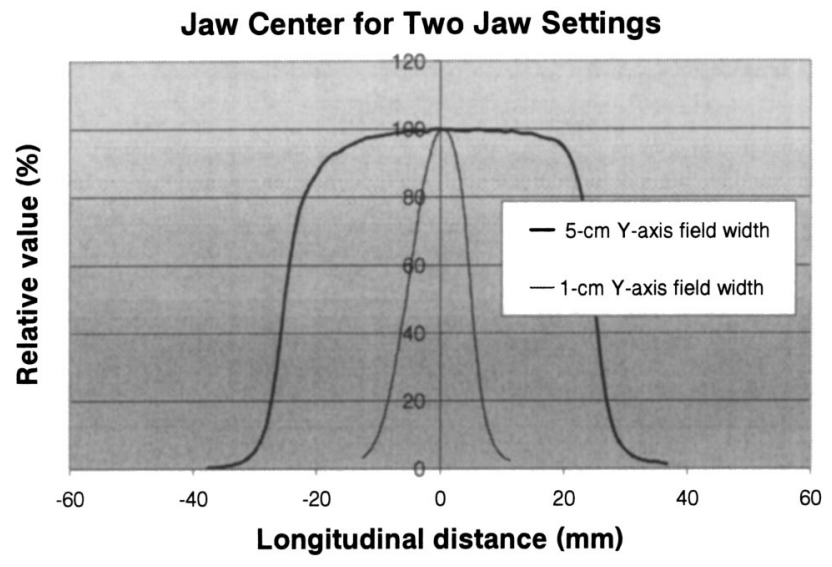

FIG. 16. This shows the profiles taken across the varying jaw setting double exposure dose image. A shift in the location of the two centers indicates an unequal motion of the jaws with respect to the calibration field positions.

cate that the image was properly rotated. The center of the narrower peaks was calculated to be halfway between the $75 \%$ dose levels. The $75 \%$ dose level was used because this was a double exposure image, and the narrower dose image ends at approximately 50\%. It was determined that there was a $0.74 \mathrm{~mm}$ shift between the two narrower peaks. The actual twist angle is calculated as follows:

Twist angle $=\tan ^{-1}$ ( shift distance/profile transverse separation distance).

This corresponds to a $0.12^{\circ}$ jaw twist for a $0.74 \mathrm{~mm}$ shift distance and a $34 \mathrm{~cm}$ profile separation distance. Assuming each measurement was accurate to within $0.1 \mathrm{~mm}$ yields an overall uncertainty of $0.02^{\circ}( \pm 0.1 \mathrm{~mm} / 0.56 \mathrm{~mm})$.

\section{MLC alignment}

A transverse profile across the $\mathrm{T} \& \mathrm{G}-\mathrm{PB}$ induced film image is shown in Fig. 18. The lower plot is the actual relative dose profile. An all-leaf-open profile is shown as well. The

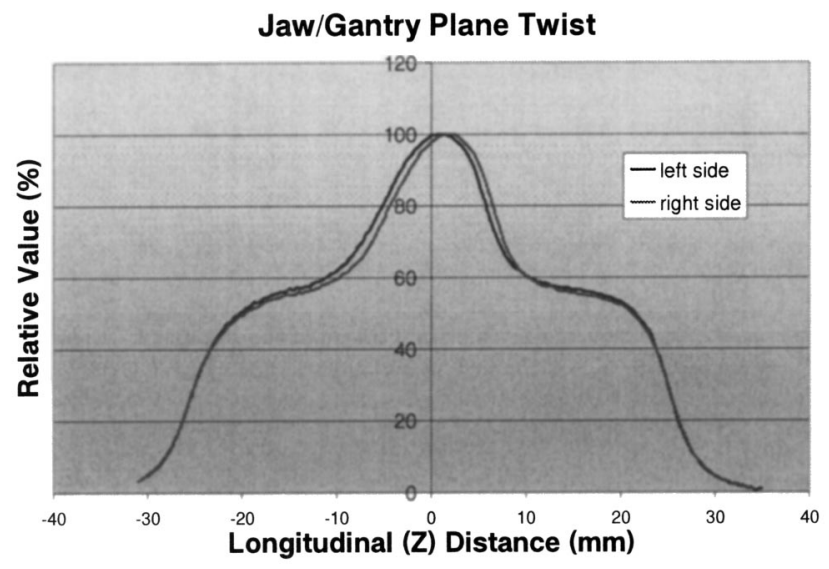

FIG. 17. This is a plot of the two profiles taken along the jaw/gantry-plane alignment dose image. The shift of the two narrow peaks is a measure of the jaw/gantry-plane misalignment.

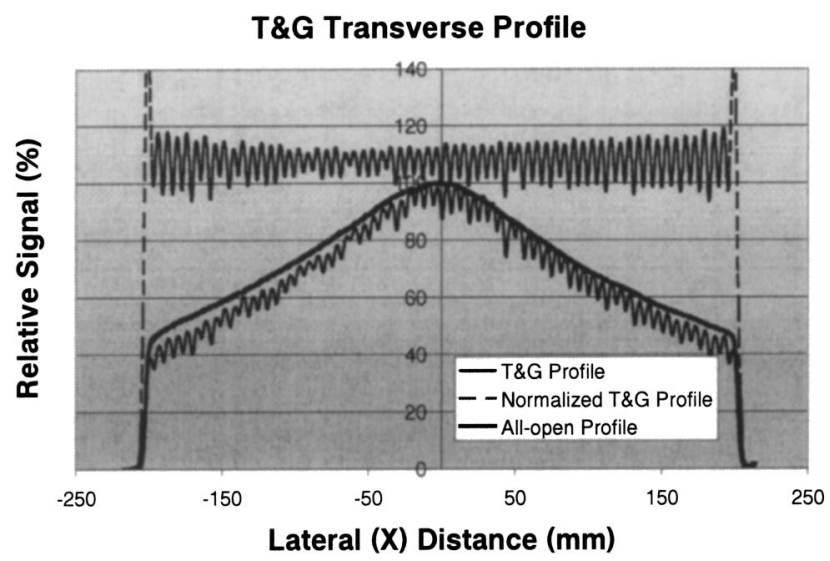

FIG. 18. This shows the transverse profile taken across the slit beam for the alternating even-leaf/odd-leaf exposure. The lower plot is the actual film profile. The upper plot is the lower plot after it has been normalized by the relative off-axis dose distribution. An all-open profile is shown as well.

T\&G-PB indications are clearly visible. A better understanding is gained by normalizing the original $\mathrm{T} \& \mathrm{G}-\mathrm{PB}$ transverse dose profile by the all-leaf-open dose profile. The allleaf-open $X$-direction dose profile has a triangle-shaped peak. The triangle shape is because the helical tomotherapy beam does not have a flattening filter. ${ }^{13}$ A measure of the MLC alignment can be inferred from the relative value of the peaks and valleys across the normalized transverse $T \& G-P B$ dose profile.

The xenon detector $\mathrm{T} \& \mathrm{G}-\mathrm{PB}$ signal profile is shown in Fig. 19. This profile was normalized by the all-leaf-open xenon-recorded signal profile. Overlaid on this plot is the normalized film-recorded T\&G-PB dose profile.

The MLC/gantry-plane twist results are shown in Fig. 20. The two T\&G-PB dips coincide exactly so there is no measurable MLC twist. (Leaf 32 open and rotated $180^{\circ}$ should produce a similar $\mathrm{T} \& \mathrm{G}-\mathrm{PB}$ artifact as a sequential opening of leaf 32 and 33.)

The dose profile across the MLC/gantry COR exposures is shown in Fig. 21. The difference between the left (leaf

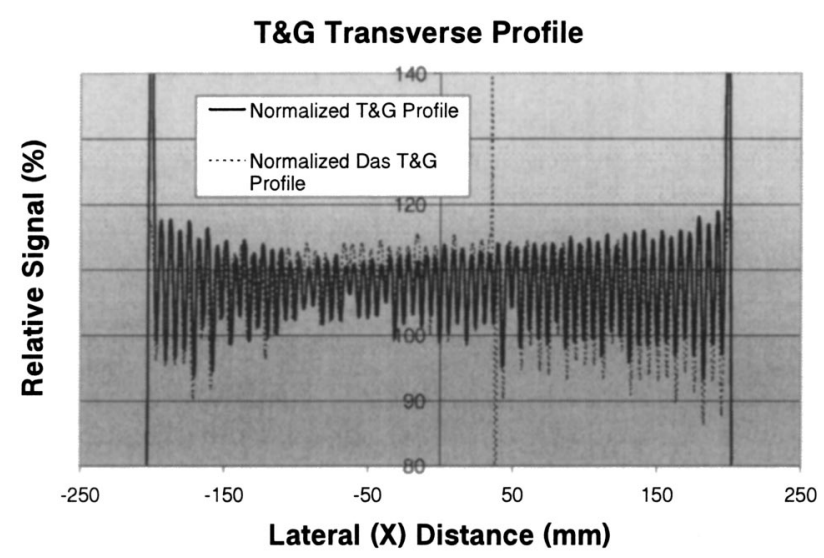

FIG. 19. The solid line is the previously shown film-recorded, normalized $\mathrm{T} \& \mathrm{G}-\mathrm{PB}$ dose profile. The dashed line is the processed and normalized $\mathrm{T} \& \mathrm{G}-\mathrm{PB}$ dose profile recorded by the xenon detectors. 


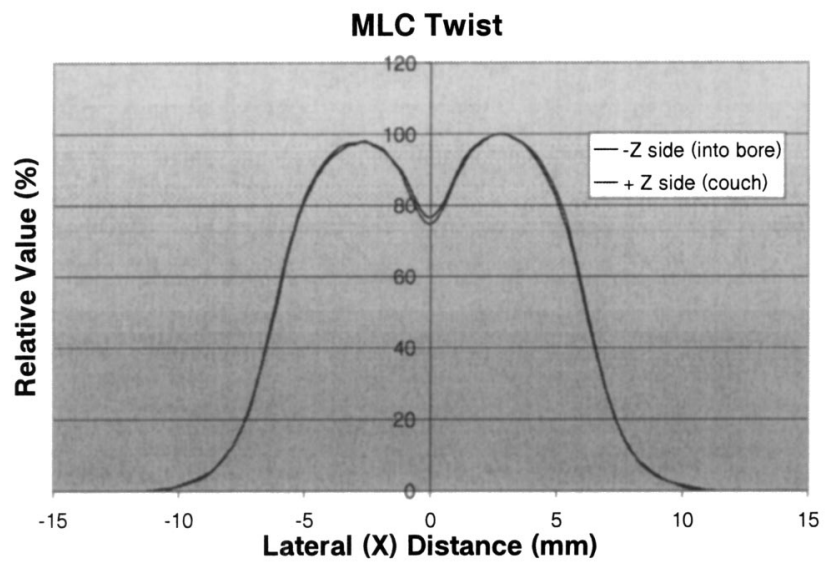

FIG. 20. This shows the dose profiles taken across the double exposure gantry-rotation leaf-32-open dose image. The shift between the two profiles is a measure of the MLC/gantry-plane twist.

28-29 gantry @ $0^{\circ}$ ) and center (leaf 32-32 gantry @ $0^{\circ}$ ) T\&G-PB dips was measured to be $25.1 \mathrm{~mm}$. The difference between the right (leaf 28-29 gantry @ 180 ${ }^{\circ}$ ) and center dip was measured to be $24.7 \mathrm{~mm}$. The assumption in this test is that the physical difference between the center of leaves 28-29 and leaves 32-33 does not change upon gantry rotation. Therefore, the difference between the left-center and center-right dips is due to the shift between the center of the MLC and the gantry COR upon rotation. The measured difference was $25.1 \mathrm{~mm}-24.7 \mathrm{~mm}$, which equals a $0.4 \mathrm{~mm}$ shift in the T\&G-PB artifact for leaves 32 and 33 upon rotation. Therefore, the region between MLC leaves 32 and 33 is offset $0.2 \mathrm{~mm}$ from the COR.

Figure 22 demonstrates the $\mathrm{T} \& \mathrm{G}-\mathrm{PB}$ dip between a sequential opening of leaves 32 and 33 upon gantry rotation. The xenon-detector recorded profiles are shown for the four static gantry angles used. The minimum fluence between the leaves is recorded at detector channel 389 for all gantry angles. This exact match for the relative shape of the profiles

\section{MLC - Center of Rotation}

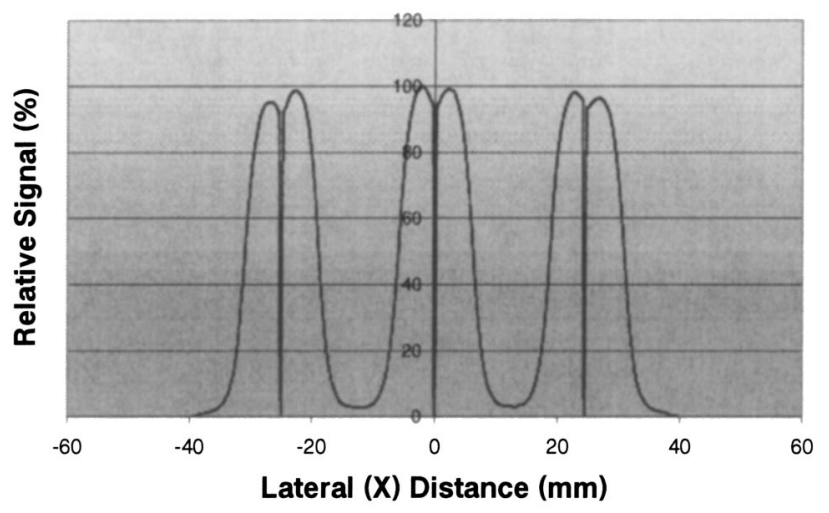

FIG. 21. This is a transverse profile with respect to the MLC/COR dose image. The relative difference between the T\&G-PB indicators denotes the position of the MLC center relative to the gantry isocenter.

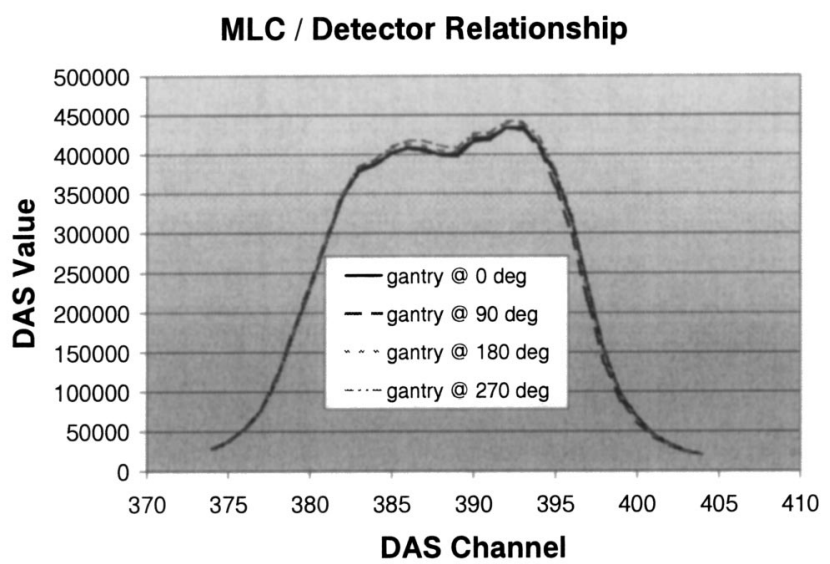

FIG. 22. This figure demonstrates the $T \& G-P B$ dip between a sequential opening of leaves 32 and 33 for the four cardinal angles of the gantry.

indicates that any effective combination of source-MLCdetector shift upon gantry rotation is well within 1 detector, which projects to $0.73 \mathrm{~mm}$ at the isocenter.

\section{Detector alignment}

The xenon-detector recorded profile for a jaw setting of $30.0 \mathrm{~mm}$ and a jaw offset of $7.5 \mathrm{~mm}$, divided by the calibration jaw setting, indicated that the xenon detectors were aligned with the jaws. The ratio at the far left side is 0.835 , and the ratio at the far right side is 0.836 . The symmetry between the left and right sides demonstrates that the xenon detector and jaws are well aligned.

\section{DISCUSSION}

Whenever possible, a double exposure film technique was used. This is advantageous in that it is relatively forgiving of film placement. The exact beam location need not be known. Moreover, these tests can be performed before the treatment couch is exactly aligned. Both of the conditions are probable during initial beam commissioning since the final couch and laser alignment are set afterwards. The film is large enough to be forgiving of small placement errors. The only restriction is that the film not be moved between the first and second exposure.

The source alignment test should be the executed fist. In this case, the test for alignment is relatively simple, but the source adjustment is relatively difficult since all the backshielding lead needs to be removed before the accelerator can be moved. This test relied on the absolute position of the jaws being accurate, i.e., the jaws are symmetrical and exactly known relative to the primary collimator center. The center-offset position is mechanically verified by an in-house alignment jig. Precise servomotors control the relative motion. This test also relied on the jaws accurately focusing on the pivot point. An erroneous source position calculation would result if they did not. The error would approximately equal the product of the angular misalignment (in radians) and the jaw rotation-point distance away from the isocenter. This could be anywhere from the proximal jaw-source posi- 
tion of $11 \mathrm{~cm}$ to the distal jaw-source position of $23 \mathrm{~cm}$. Mechanical engineering analysis indicates that a jaw/pivotpoint misalignment error greater than $0.1^{\circ}$ is not likely. Values less than that would not significantly alter the results. A $0.1^{\circ}$ error about a rotation point halfway along the length of the jaws $(17 \mathrm{~cm}$ for the source position) would shift the jaw-focus point $0.3 \mathrm{~mm}$. This would perturb, but not radically alter the results as the jaws sweep a distance of $2.5 \mathrm{~mm}$ at the source plane.

The source location results presented here relied simply on the peak intensity signal. The response could easily be fit to a parabolic curve for better statistical results, if needed. However, since the solution to any misalignment is to move the accelerator, it is not felt that such statistical accuracy is warranted.

The MLC/source alignment is more easily adjusted by moving the source rather than the MLC. Any recommended source adjustment would be in the $X$ direction, but it would be prudent to recheck all source and jaw alignment parameters. The clinical physicist may wish to repeat some of the rotational tests described herein for the gantry at $0^{\circ}$ and $180^{\circ}$ for lateral gantry angles, and would be encouraged to do so. The principal is the same. The detector alignment test presented was only done with the gantry at $0^{\circ}$. This also could be repeated at the four cardinal angles, if desired. The UW unit showed no appreciable difference at those angles, but it is possible that the accelerator structure could be perturbed upon gantry rotation, and thus affect the detector/jaw alignment.

The $Y$-axis misalignment of the beam center is adjusted by shifting offsets of the moveable jaws relative to their nominal offset positions. This is a very simple software parameter input value, as long as the source is approximately centered within the primary collimator. The next test presented checked if the moveable jaws moved equally and opposite from the position at which they were set to have no $Y$-axis misalignment. It is reassuring if they do, but as long as any discrepancy is submillimeter, it will not matter clinically as it can be dealt with separately in planning. An unacceptable jaw twist with gantry rotation value would be difficult to correct, but could if need be. The MLC and other components would have to be removed beforehand.

The MLC/source alignment is an area that involved multiple source adjustments. The initial test showed a distinct MLC/source misalignment. This was made evident by the initial normalized T\&G-PB transverse profile (not shown). A careful accelerator alignment in the $X$ direction has been shown not to affect the previously adjusted alignment in the $Y$ direction. However, the opposite was also shown for less careful adjustments. Therefore, when the accelerator is adjusted for MLC alignment, the $Y$-axis misalignment tests should be repeated. The MLC/source alignment test produced the only results that were not quantified. The MLC was considered sufficiently aligned via a visual inspection of the T\&G-PB profile. The magnitude of the T\&G-PB peaks and valleys are approximately equal. The symmetry at the far lateral edges is especially important for alignment determination. An automatic analysis tool is made more difficult by the random size of the T\&G-PB effects between ( equally focused) adjacent leaves due to random machining/assembly variations. Nevertheless, an automatic analysis tool is under development.

The MLC/gantry-plane alignment would be a difficult parameter to fix, but it is not likely to be in error. This is likewise for the MLC/xenon detector consistency. Both the MLC and the detectors are fastened to the same high precision CT gantry, so it is not likely there would be an inconsistency between them upon rotation. Regardless, like all alignment parameters, it needs to be verified.

The MLC/gantry COR results just need to be quantified. The optimization/dose calculation code is capable of optimizing with an MLC/COR offset.

The post-patient xenon-filled 762 element detection array proved very useful. It was able to assist in its own alignment verification, both for its consistent mapping with the MLC leaves, and for its alignment with the plane of gantry rotation. It was also shown to be able to adequately replace film for the MLC/source alignment test. The spatial resolution of the detectors was almost as fine as digitized film results, as determined by the similarity between the transverse profiles in Fig. 18, for measuring the magnitude of the T\&G-PB effects. Any test that can be done via the xenon detectors can be automated and the results can be known in nearly real time.

\section{CONCLUSION}

The first clinical helical tomotherapy machine has been installed and commissioned at the University of Wisconsin. The accelerator $\mathrm{x}$-ray source and the beam-collimation devices are well aligned. The tests for verifying and quantifying this were presented. Very little specialized equipment is required. Radiographic film is the predominate dosimeter used. A single specialized external ion chamber was used for one alignment test. The Hi-ART system provides a postpatient megavoltage xenon-detector array that has proved useful for many alignment tests.

TomoTherapy Incorporated has written software for facilitating the analysis of film and xenon-detector data. Any test that can be performed with the xenon-detector data can be automated and analyzed quickly.

\section{ACKNOWLEDGMENTS}

This work was funded by TomoTherapy Inc. and by NIH PO1 CA088960.

\section{APPENDIX: HELICAL TOMOTHERAPY CALIBRATION DETAILS}

The Task Group 51 protocol specifies the calibration procedure for therapeutic megavoltage radiation beams. ${ }^{14}$ It explicitly specifies a setup of a $100 \mathrm{~cm} \mathrm{SSD} \mathrm{and} \mathrm{a} \mathrm{percent}$ depth dose measurement at $10 \mathrm{~cm}$. The maximum tomotherapy field size is $5 \mathrm{~cm}$ by $64 \mathrm{~cm}$ at the isocenter, which is $85 \mathrm{~cm}$ away.

The couch is lowered by its maximum distance, $27 \mathrm{~cm}$ and a water tank is filled with $12 \mathrm{~cm}$ of water. This sets the 
SSD to $100 \mathrm{~cm}$. A chamber can be set to a depth of $10 \mathrm{~cm}$, and still have more than $2 \mathrm{~cm}$ of backscatter (when including the couch). The central 40 leaves were set open. Each leaf projects to 6.25 millimeters in the transverse direction at the isocenter, so the overall transverse distance is $29.4 \mathrm{~cm}$ (at $\mathrm{SSD}=100 \mathrm{~cm}$ ). The $Y$-axis field width at $100 \mathrm{~cm} \mathrm{SSD} \mathrm{is} 5.9$ $\mathrm{cm}$. This equals an equivalent field size of approximately 10 $\mathrm{cm}$ by $10 \mathrm{~cm}$ specified by the product of the $X$-axis dimension and the $Y$-axis dimension divided by the perimeter of the rectangular field. ${ }^{15}$ The required TG51 protocol parameter $K_{q}$ is then determined from the percent dose value measured at $10 \mathrm{~cm}$ versus that measured at the depth of maximum dose. This computed $K_{q}$ value, along with the other TG51 correction factors is then used to determine the output for the $5 \mathrm{~cm}$ by $10 \mathrm{~cm}$ calibration field at the depth of maximum dose at the isocenter.

The authors realize that the equivalent-square field size approximation breaks down at highly rectangular field sizes, but the $K_{q}$ correction factor is typically close to unity for a 6 MV linear accelerator. The depth $10 \mathrm{~cm}$ percent depth dose for the $100 \mathrm{~cm}$ SSD field was $63.0 \%$. The $K_{q}$ value for this energy for the A1SL ion chamber (Standard Imaging, Middleton, WI) is 0.999 . The depth at $10 \mathrm{~cm}$ percent depth dose value for the $85 \mathrm{~cm} \mathrm{SSD} \mathrm{field} \mathrm{was} \mathrm{60.8.} \mathrm{The} K_{q}$ value for this energy is also 0.999 . Therefore, it is unlikely that the use of an equivalent square correction factor produces an error greater than a few tenths of a percent.

More research, employing Monte Carlo analysis, specifically addressing the recommended calibration technique for helical tomotherapy is ongoing.

${ }^{1}$ T. Mackie, T. Holmes, S. Swerdloff, P. Reckwerdt, J. Deasy, J. Yang, B. Paliwal, and T. Kinsella, "Tomotherapy: A new concept for the delivery of conformal radiotherapy," Med. Phys. 20, 1709-1719 (1993).

${ }^{2}$ T. R. Mackie, J. Balog, K. Ruchala, D. Shepard, S. Aldridge, E. Fitchard, P. Reckwerdt, G. Olivera, and T. McNutt, “Tomotherapy," Semin Radiat. Oncol. 9, 108-117 (1999).
${ }^{3}$ D. A. Low, K. S. Chao, S. Mutic, R. L. Gerber, C. A. Perez, and J. A. Purdy, "Quality assurance of serial tomotherapy for head and neck patient treatments," Int. J. Radiat. Oncol., Biol., Phys. 42, 681-692 (1998). ${ }^{4}$ D. A. Low, S. Mutic, J. F. Dempsey, J. Markman, S. M. Goddu, and J. A. Purdy, "Abutment region dosimetry for serial tomotherapy," Int. J. Radiat. Oncol., Biol., Phys. 45, 193-203 (1999).

${ }^{5}$ J. N. Yang, T. R. Mackie, P. Reckwerdt, J. O. Deasy, and B. R. Thomadsen, "An investigation of tomotherapy beam delivery," Med. Phys. 24, 425-436 (1997).

${ }^{6}$ C. S. Chui, S. Spirou, and T. LoSasso, "Testing of dynamic multileaf collimation," Med. Phys. 23, 635-641 (1996).

${ }^{7}$ M. Essers, M. de Langen, M. L. Dirkx, and B. J. Heijmen, "Commissioning of a commercially available system for intensity-modulated radiotherapy dose delivery with dynamic multileaf collimation," Radiother. Oncol. 60, 215-224 (2001).

${ }^{8}$ C. B. Saw, K. M. Ayyangar, R. B. Thompson, W. Zhen, and C. A. Enke, "Commissioning of Peacock System for intensity-modulated radiation therapy," Med. Dosim 26, 55-64 (2001).

${ }^{9}$ E. E. Klein, D. A. Low, J. W. Sohn, and J. A. Purdy, "Differential dosing of prostate and seminal vesicles using dynamic multileaf collimation," Int. J. Radiat. Oncol., Biol., Phys. 48, 1447-1456 (2000).

${ }^{10}$ V. P. Cosgrove, U. Jahn, M. Pfaender, S. Bauer, V. Budach, and R. E. Wurm, "Commissioning of a micro multi-leaf collimator and planning system for stereotactic radiosurgery," Radiother. Oncol. 50, 325-336 (1999).

${ }^{11}$ J. M. Galvin, A. R. Smith, and B. Lally, "Characterization of a multi-leaf collimator system," Int. J. Radiat. Oncol., Biol., Phys. 25, 181-192 (1993).

${ }^{12}$ J. Balog, T. R. Mackie, L. Angelos, and P. Reckwerdt, "Dose computation for a one dimensional multileaf collimator," Proceedings from the XII International Conference on the use of Computers in Radiation Therapy (Medical Physics Publishing, Madison, WI, 1997), pp. 434436.

${ }^{13} \mathrm{~J}$. Balog, "Commissioning dosimetry for a clinical tomotherapy helical accelerator," Proceedings Biological \& Physical Basis of IMRT \& Tomotherapy (Medical Physics Publishing, Madison, WI, 2001), pp. 88-93.

${ }^{14}$ P. R. Almond, P. J. Biggs, B. M. Coursey, W. F. Hanson, M. S. Huq, R. Nath, and D. W. Rogers, "AAPM's TG51 protocol for clinical reference dosimetry of high-energy photon and electron beams," Med. Phys. 26, 1847-1870 (1999).

${ }^{15} \mathrm{~F}$. Khan, The Physics of Radiation Therapy, 2nd ed. (Williams and Wilkins, 1994), Chap. 9, p. 184. 